\title{
LA EXPERIENCIA DE SUFRIMIENTO EN LOS TRASTORNOS ALIMENTARIOS: HACIA UN MODELO INTEGRATIVO
}

\author{
DIANA KIRSZMAN y M. CARMEN SALGUEIRO
}

Fundación Aigle, Buenos Aires

(Aceptado en febrero de 2001)

Los trastornos alimentarios, como ejemplo paradigmático de patología multidimensional, plantean un desafío que exige del clínico el despliegue de diversos abordajes y técnicas. Pero no basta sumar recursos, es necesario integrarlos, saber cuándo y cómo intervenir y con qué criterios. Las consultas pueden adoptar distintas modalidades de demanda y organizarse en representaciones de la experiencia disfuncionales, cuya gravedad se ubica a lo largo de un continuo amplio: desde la simple preocupación por la dieta y la figura, hasta las situaciones de enfermedad plenamente desarrollada. Se ha dicho que los trastornos alimentarios constituyen un fracaso, o una amenaza, para la autonomía. Hoy sabemos que las estructuras de significado afectadas recorren todo el espectro evolutivo, desde las etapas tempranas que involucran al cuerpo y sus funciones perceptivas y motrices, hasta las más tardías que reclaman el compromiso con otro. En este sentido, aún cuando la adolescencia continúa siendo la edad de mayor riesgo, estas patologías han trascendido sus límites. La psicoterapia, desde un modelo cognitivo, entendida como un ejercicio del lenguaje, y en armonía con otros recursos terapéuticos, se proyecta como un instrumento privilegiado para la resignificación y el cambio de conducta.

Palabras clave: Trastornos alimentarios, integración, modelo cognitivo, estructuras de significado, psicoterapia.

The distressing experience in the eating disorders: towards an integrative model

Eating disorders, as a paradigmatic example of multidimensional pathology, put forward to the clinic the challenge of knowing, and therefore using, different approaches and techniques. But it is not an issue of adding up different assets: integration is mandatory in order to recognize how to intervene and the criteria to do it accurately. Consultation is structured in different ways for different people; the demand for treatment or help is organized around dysfunctional representations of experience. Severity runs along a broad range, goes from the simple preoccupation about dieting, weight and corporal figure to the full expression of the traditional diseases: anorexia or bulimia. Eating disorders have been endorsed to failures in autonomy. We know today that meaning structures affected in eating pathology involved the whole evolutive continuum, from early stages dealing with body functions and their perceptive and motion capacities to late stages having to do with engagement and commitment to relationships with others. So, even though adolescence is still the most affected period, eating defined as a language endeavor and in harmony with other therapeutic resources, is a privileged instrument for changes in meaning and conduct.

Key words: Eating Disorders, integrative, cognitive models, meaning construction, psychotherapy

INTRODUCCIÓN: ¿POR QUÉ INTEGRAR?

El tratamiento de los trastornos alimentarios, así como el de muchas otras situa-

Correspondencia: FUNDACIÓN AIGLE. Virrey Olaguer y Feliú 2679, 1426 Buenos Aires, Argentina. Correo-e: faigle@teletel.com.ar ciones clínicas, implica el despliegue de una gran diversidad de abordajes y técnicas. La multiplicidad de factores implicados: biológicos, psicológicos y contextuales, no acepta conceptualizaciones únicas y dogmatizadas. Los modelos multidimensionales, y particularmente aquellos que integran lo teórico y lo prác- 
tico, que ofrecen estructuras que tratan de dar respuestas tanto a escala conceptual como a nivel observacional y clínico, son aquellos que están en mejores condiciones de abarcar la complejidad en la presentación de la patología alimentaria. El modelo con el que trabajamos intenta abarcar esta complejidad desde una perspectiva integradora. Pero ¿qué quiere decir integrar?

El término integración proviene de íntegro, que significa entero, lo que a su vez implica algo sub-divisible, constituido por distintas partes. Aquello que se intenta integrar son enfoques, modos de resolución de aspectos parciales, pero que han resultado útiles en la explicación y abordaje de determinado fenómeno o problema. El concepto de integración no tiene que ver con algo único, sino que supone dispersión. Para que algo pueda ser entero, íntegro, necesita partes que lo compongan. Asimismo, al hablar de integración y no de unión, lo esperable que suceda en el desarrollo clínico futuro es que esa integración se desintegre para ir dando lugar a integraciones sucesivas de distintos niveles de complejidad. Nunca se piensa la integración como algo totalizador, de una vez y para siempre, sino de sintesis sucesivas. Tampoco existe una única manera de integrar, sino muchas. La que proponemos es una de ellas y, en este sentido, ninguna de las palabras usadas en la denominación es azarosa. Cuando hablamos de «un» modelo, en realidad queremos decir que hay muchos, y que este que proponemos es uno entre muchos posibles y no «el» único modelo de integración que pueda proponerse. Es el que hasta ahora nos ha resultado de utilidad en la evaluación y tratamiento de una amplia diversidad de pacientes y situaciones de consulta y, aún así, como decíamos, está en permanente revisión.

Cuando pensamos en las situaciones de consulta resulta de utilidad clasifi- carlas en alguna de las siguientes tres categorías: pueden estar centradas en el organismo, pueden estar vinculadas a algún aspecto del desarrollo personal, y pueden ser expresiones de un malestar en la comunicación. En este sentido, los trastornos alimentarios constituyen un ejemplo paradigmático, puesto que la experiencia de padecimiento que los caracteriza incluye, en mayor o menor medida, todas y cada una de estas tres categorías. En primer lugar, es un malestar centrado en el cuerpo, el cuerpo se instaura en fuente de experiencias de malestar y sufrimiento. En segundo lugar, en los adolescentes, la población más afectada, expresa algún obstáculo en el desarrollo del guión personal, por la dificultad para construir significados que sostengan una experiencia autónoma. Por último, en gran parte de su manifestación y gravedad influyen las dificultades en la comunicación, expresadas a través de alguna o varias disfunciones en los vínculos más significativos. La imagen corporal de las pacientes se carga de significados, desde la insatisfacción hasta, en los casos extremos, verdaderas distorsiones que conducen a prácticas extremadamente agresivas y auto-destructivas para "corregirla" - ayunos, ejercicios extenuantes, cirugías, etc.- - La búsqueda de un ideal de belleza, finalmente inalcanzable para la mayoría de las mujeres, se instala en el núcleo central del proyecto personal, inundando el universo propio y arrasando con otros intereses y metas: el guión personal es tomado por asalto. Las pacientes se aíslan, se esconden, se alienan de sus vínculos más cercanos.

En toda situación de consulta hay alguien que demanda y alguien que oferta, y ambas, demanda y oferta, son expresión de un marco socio-cultural específico. La demanda tiene una estructura y una fuente de derivación propias, y se manifiesta en diversas formas de males- 
tar que atraviesan a quien consulta. La oferta también tiene una estructura determinada, un marco socio-profesional específico; $y$, en ella, el elemento más complejo está dado por las cualidades particulares y el estilo personal del terapeuta que tome a su cargo el proceso terapéutico. En condiciones óptimas, la evaluación del proceso terapéutico dará como resultado un alto nivel de satisfacción en los protagonistas implicados, no sólo en quien o quienes demandan ayuda, sino también en quien o quienes la ofrecen.

Quienes buscan y demandan ayuda son personas que padecen un malestar específico y la experiencia de ese padecimiento tiene siempre una representación personal. Decimos persona y no individuo. Persona puede ser un individuo o más de un individuo. Se refiere a una cuestión personal y, por lo tanto, no es algo relativo a un orden institucional, como sería «la» institución familia, por ejemplo. Aún cuando trabajemos con familias, el destinatario del acto terapéutico, a diferencia de los modelos sistémicos, no es la familia "en sí» sino las personas que la integran. El análisis de la demanda de ayuda es un elemento fundamental en nuestro modo de acercarnos a las situaciones de malestar y, particularmente, a las vinculadas a los trastornos de la alimentación y de la imagen corporal. Para nuestro modelo resulta central la consideración de su constitución, de su historia, de sus componentes. En algunas ocasiones, la demanda es clara, está madura y es fácil seguir su recorrido e identificar los componentes que la conforman. En otras, la demanda de ayuda es confusa; resulta difícil identificar los núcleos del malestar, sus orígenes y los caminos que ha recorrido. La demanda puede ser múltiple y organizarse en representaciones individuales de varios miembros de un grupo familiar. Estas representaciones no siempre son concordantes. En este sentido, el trabajo sobre estos aspectos de la demanda de ayuda, su tallado y conformación constituye el primer objetivo del acto terapéutico.

¿Quién o quiénes se ubican en el otro polo de este acto terapéutico? Puesto que se trata de trastornos de causalidad y forma de presentación múltiple, un equipo multidisciplinario. Esto es esencial. Aún en los casos más leves, la intervención psicológica individual no es suficiente, y es necesario realizar, por ejemplo, algún tipo de evaluación, asistencia y seguimiento médico-nutricional. Y es más: en los casos graves es tal el nivel de demanda y de presión que se ejerce sobre el equipo terapéutico, tal la complejidad en la toma de decisiones durante el tratamiento - manejo de crisis, complicaciones médicas que ponen en riesgo la salud y la vida, etc.- - que el trabajo solitario se hace prácticamente imposible. Y si no imposible, al menos, arriesgado.

El malestar específico es una cierta forma de padecer, la que será objeto de actos terapéuticos. En la manera en que se entienda esta particular forma de padecer, comienza a jugar un papel importante lo integrativo, ya que son varios los determinantes que se entrecruzan en la misma. En primer lugar, decimos que el padecimiento tiene un determinante histórico: se ha organizado históricamente; por lo tanto, requiere estar motivado. $O$, lo que es lo mismo, nadie sufre porque sí: no existe sufrimiento que no tenga una historia que lo justifique. En segundo lugar, este sufrimiento tiene que tener una circunstancia presente que lo recuerde: necesita de algo que opere en el momento de la consulta, que lo actualice; si no, se extinguiría. Es decir, una experiencia humana necesita por un lado una organización histórica que la constituya, que le de origen, que la motive y, por el otro, circunstancias actuales que la recuerden, que la refuercen, que la sos- 
tengan. En tercer lugar, para que alguien sufra, es necesario que exista una cierta disposición, ya sea en uno de los miembros de la familia o en varios. Esto tiene que ver con los factores de vulnerabilidad individual y/o familiar, que hacen que las personas se enfermen o padezcan de una manera y no de otra. Por último, para que los individuos puedan realizar esta disposición, es decir, volverla concreta y material, es necesario que exista un contexto en el cual esa disposición se exprese y actualice. Este contexto, además, contribuye a sostenerla, mantenerla, reforzarla, etc. Aquí es donde entran en consideración los contextos familiares y los contextos sociales amplios como elementos fundamentales a tener en cuenta en los factores etiopatogénicos de las distintas condiciones clínicas.

Para poder decir que integramos, y no simplemente que sumamos técnicas o abordajes, es necesario encontrar perspectivas desde las cuales se pueda entender la realidad de ese padecimiento movilizante de ayuda. Y para entender $y$ abordar dicha realidad no es suficiente situarse en alguna o más de las cuatro atalayas mencionadas, esto es, ni en el nivel de la determinación histórica, ni en el de la manera en que el presente condiciona, ni en el del individuo, ni en el del contexto, sino desde otra perspectiva en la que estas cuatro cosas sean percibidas en el mismo nivel. Esta visión privilegiada es epistemológicamente congruente, no vulnera los principios generales de la integración en psicoterapia. Hay perspectivas que observan lo que ocurre en la situación clínica atendiendo a lo que les sucede a las personas, $\mathrm{y}$ hay perspectivas que atienden a lo que les ocurre a los grupos, a los sistemas, a las familias. Pero hay una tercera perspectiva que observa un punto intermedio, que no es ni el individuo, ni el sistema, ni la familia: este punto de observación intermedio es el de las funciones interactivas. Cuando uno observa la interacción, no observa ni el individuo ni el sistema, observa la forma particular en que dos o más personas se relacionan. Lo mismo ocurre con lo histórico y lo actual, con la función motivadora y la función reforzadora. Existen modelos o teorías que enfatizan la importancia de los motivos, la historia, los hechos del pasado que determinan activamente el presente y existen modelos o teorías que focalizan los factores de aprendizaje y de refuerzo en el momento actual. En el encuentro con una persona que padece, que está deprimida, por ejemplo, uno puede, desde una perspectiva, ver cuáles son las circunstancias que llevaron a que esté deprimida y ver, desde otra perspectiva, cuáles son las circunstancias que hacen que siga estando deprimida. Pero, desde una mayor distancia, es posible una tercera perspectiva, un punto de observación privilegiado desde el cual se pueden ver las dos cosas simultáneamente. Podemos tener esa perspectiva haciendo un análisis de las construcciones de significado con las que la persona que padece organiza cognitivamente su experiencia.

La experiencia puede definirse como el conjunto de operaciones internas y externas que realizan los seres humanos movidos por su afán de conocimiento, de ahí su carácter cognitivo. Lo que moviliza permanentemente la experiencia es la búsqueda de conocimiento. La experiencia es, además, el entrecruzamiento de la actividad biológica, la actividad psicológica y la actividad social. Estos tres elementos ocurren simultáneamente, ninguno de ellos puede privilegiarse sobre los restantes. Tiene, finalmente, un carácter constructivo: la realidad en la que los seres humanos despliegan su actividad debe ser conocida, organizada; y la organización es una tarea constructiva.

Constituye un error común considerar que las cogniciones tienen solamente una 
fuente intelectual. Por el contrario, lo intelectual es sólo una de las fuentes de lo cognitivo. Permite organizar los aspectos formales y de contenido de la experiencia por medio de organizaciones lógico-formales y, también, por medio de la creatividad. Pero lo cognitivo no se agota en este aspecto intelectual. Otra fuente de lo cognitivo está constituida por lo afectivo, tanto en sus aspectos expresivos como intensivos. Lo afectivo hace referencia a la organización de aquellos aspectos de la experiencia que traducen el caudal de energía y las cualidades de las fuerzas que operan en el realidad. Incluye, además, lo evaluativo, aquello que se refiere a la capacidad de organizar la experiencia de acuerdo con significados valorativos, con reglas y normas disponibles para cada individuo y cada contexto social determinado. Lo evaluativo constituye el orden ideológico de la constitución de nuestra realidad. Al mismo tiempo, debe descartarse la idea de que los procesos de conocimiento de la realidad responden exclusivamente a la actividad consciente. Los procesos inconscientes ocupan un lugar primordial en la acción de conocer, son procesos muy significativos que operan ininterrumpidamente.

\section{LA EXPERIENCIA DISFUNCIONAL}

Como personas, estamos inmersas permanentemente en un universo cargado de estímulos e informaciones. Estímulos que debemos percibir, atender, clasificar $y$, finalmente, significar. Necesitamos organizar nuestra experiencia personal, la forma en que nos representamos el mundo y la forma en que nos representamos a nosotros mismos dentro de ese mundo. Esta organización personal de todo el cúmulo de información que nos rodea se expresa a través de construcciones de significado, formas de organiza- ción de la experiencia personal en la que cada uno refleja su representación del mundo, incluyendo su propia auto-representación.

Existe una relación recíproca entre estas dos características, lo cognitivo y lo constructivo: conocemos porque somos capaces de construir una representación de la realidad que nos rodea, y construimos nuestra realidad porque somos capaces de conocer. Del interjuego entre lo cognitivo y lo constructivo, surge una organización: dicha organización se expresa en un sistema integrado por estructuras de significado, que tienen dos características fundamentales. En primer lugar, son operaciones necesarias, puesto que a todos se nos hace necesario organizar y manejar nuestro mundo de información, seleccionar, ordenar, clasificar, resolver, etc. A veces lo hacemos bien, otras veces no, y esto nos produce cierto sufrimiento. Y, en segundo lugar, estas operaciones están dirigidas a sostener el sentimiento de identidad personal, puesto que los mundos que vamos creando son mundos en los que nos colocamos, vivimos en ellos. El ser humano, como activo constructor de experiencia y en su afán por dotar de significado a la información, tanto interna como externa, a la que se encuentra expuesto, va creando constructivamente mundos dentro de los cuales puede ubicar su propia identidad. Hay mundos que le permiten vivir bien y construir su experiencia adecuadamente, ya que fundan un buen alojamiento para la identidad; $y$, por otro lado, hay mundos generadores de malestar y sufrimiento. Veamos un ejemplo:

Lili se aloja desde sus 20 años y sus $38 \mathrm{Kg}$. en uno de estos mundos. Desde su cosmovisión, el éxito y el control son las únicas alternativas posibles. Se las ingenió para lograr siempre lo que quiso en materia de rendimiento: la estudiante perfecta. Sus preocupaciones se organizan alrededor de dos temas: una es su peso. Nunca pudo estar lo 
suficientemente flaca ni lo suficientemente atractiva. La otra, es su necesidad de satisfacer a los demás, particularmente a sus padres. Cada uno de sus actos está al servicio de estos objetivos. Ávida, supervisa día a día su ambiente a la búsqueda de señales de reconocimiento, un gesto afectuoso de su madre que se destaque entre los que dispensa a sus hermanas, o una palabra de su padre robada entre el celular y la oficina. Uno solo de estos signos puede marcar la diferencia entre el cielo y el infierno de ese día; el resto es ayuno y extenuación...

Este ejemplo, así como muchas de las experiencias personales que se traducen en situaciones de consulta específica, revela construcciones de significado que han organizado de manera disfuncional la experiencia personal con relación al self y al mundo circundante. La representación personal se organiza en significados funcionales cuando es fuente de sentimientos de bienestar o felicidad: la persona siente que su identidad individual es fuerte; se siente segura en cuanto sus raíces, sólida; experimenta una sensación de consonancia entre su propia experiencia y la de los demás; se siente confirmada, convalidada; su auto-evaluación es positiva, etc. Por el contrario, cuando la experiencia no se organiza adecuadamente, la identidad se aloja en un mundo inadecuado. Esto puede suceder por distintas razones, pero lo que se genera siempre es un sentimiento de malestar y desequilibrio. La identidad se vivencia débil, insegura, con grietas; las relaciones con los otros se vuelven disonantes, problemáticas o conflictivas; la auto-evaluación es predominantemente negativa, etc.

En general, podemos decir que es posible organizar adecuadamente la experiencia cuando se le puede otorgar algún significado, siempre que podamos generar un proyecto articulado entre los mandatos precedentes y la trayectoria propia, y siempre que sea posible equilibrar las necesidades de estabilidad con las necesidades de cambio. Este plano psicológico, se aúna con los principios organizadores del orden de lo biológico, que aseguran el funcionamiento vital; y con los del orden social, que tienen que ver con el funcionamiento de las instituciones, su normativa y su legalidad. Los tres coexisten posibilitando en el ser humano una experiencia integradora. Cuando las personas dicen no sentirse bien, cuando se sienten infelices, cuando creen que su vida no es la que planificaron, cuando viven el futuro de forma amenazadora, sufren; y al no encontrar sentido a su sufrimiento, éste pasa a constituirse en representaciones negativas de la realidad y de sí mismas, y en la expresión de alguna forma de malestar, alteración, trastorno, desorden, enfermedad, perturbación, disturbio, etc. Existe malestar cuando no se puede asignar un significado a la experiencia, por ejemplo, en los trastornos graves que se expresan en estados confusionales, amenazas o intentos suicidas, o en las múltiples manifestaciones de las psicosis. Existe malestar cuando se verifica una discordancia entre el guión parental y el guión personal y esta discrepancia no puede ser resuelta, negociada o elaborada de alguna manera. También existe malestar cuando hay un marcado desequilibrio entre los procesos que tienden a consolidar la identidad personal y aquellos procesos que tienden a mover a las personas hacia un lugar de mayor complejidad o madurez. Estos dos últimos aspectos - la articulación de los guiones personal y parental y el equilibrio entre los procesos de estabilidad y cambio- son los más específicamente vinculados con el quehacer de la terapia familiar.

La tarea constructiva para dotar de significado a la experiencia se da en forma evolutiva, atraviesa distintas etapas a lo largo de la vida. En cada una de estas etapas es necesario dar respuesta a deman- 
das, tanto de orden biológico, como psicológico y social. Las distintas construcciones de la identidad personal están ordenadas jerárquicamente y aparecen evolutivamente en el siguiente orden: función discriminante básica, filiación y parentesco, dominación, diferenciación sexual, reconocimiento, autonomía, transitividad, productividad y perdurabilidad, y recogimiento. No es este el momento, ni el lugar, para desarrollar cada una de estas estructuras en detalle, pero pasaremos revista a algunos de los aspectos más significativos, y especialmente los vinculados con los trastornos alimentarios.

\section{LAS CONSTRUCCIONES DE SIGNIFICADO}

En el caso de los trastornos alimentarios, y según nuestra experiencia, hemos encontrado dificultades, desde las etapas más tempranas hasta las más tardías, en las construcciones de discriminación, dominio, diferenciación sexual, reconocimiento y autonomía. Recordemos algunas características de la primera construcción tal como está descrita por Fernández-Álvarez (1992):

La primera construcción, la función discriminante básica, se organiza sobre la base del desarrollo de estructuras de significación vinculadas a los límites corporales (internos y externos), comenzando por la elaboración de protoesquemas anatómicos y funcionales. Ello es posible gracias a la elaboración de formas primitivas de significar las primeras experiencias de intercambio con el entorno, directamente asociadas a los estados de necesidad y saciedad (o no) de los requerimientos planteados por dichos estados. Oxigenación, alimentación, descanso, son algunas de las acciones que marcan los primeros intercambios constitutivos de la experiencia.... Basado en la estructuración de significados que definen sus experiencias concretas de separación del cuerpo materno, de diferencias térmicas, lumínicas, auditivas, etc., de estima- ciones sobre el ritmo de los acontecimientos medioambientales y del tiempo transcurrido entre la necesidad y la saciedad,... el cumplimiento de la satisfacción de sus necesida. des, etc.

Esta estructura es la que da lugar a la constitución inicial de un individuo a partir de la discriminación entre lo propio y lo ajeno. Sienta las bases para la constitución de una identidad personal que luego habrá de complejizarse evolutivamente con el desarrollo de las otras estructuras. La importancia que cobra en esta etapa la integridad de todos los sistemas físicos y sensoriales, y la cualidad de la relación entre el recién nacido y sus padres se verifica en muchas situaciones clínicas, los trastornos alimentarios entre otras.

Comenzamos a tener datos experimentales que muestran la influencia de los modos de comportamiento con respecto a la comida en las relaciones tempranas. Estos datos permiten inferir que aspectos experienciales de la maternidad y la ingesta están íntimamente relacionados. De hecho, se verifica que una madre que observa en su bebé el rechazo del alimento, se angustia significativamente. Comienza un círculo de desconcierto, de impotencia y, en algunas situaciones, de desequilibrio. Esto puede conducir a la madre a adoptar una actitud sobreprotectora que es difícil de cambiar con el paso del tiempo, aún cuando la conducta hacia la comida, en el hijo, se modifique. Es frecuente encontrar, entonces, que madre e hija, o hijo, forman una díada potenciadora de malestar, que está guiada por cuánto come, cómo come, etc. En el mundo animal, una madre que verifica que una de sus crías no come, es presa de ansiedad. Algo le dice que existe un peligro en la cría. En cambio, cuando la cría agarra el pezón, este gesto produce una sensación automática de tranquilidad. 
Orbach (1985), desde su perspectiva feminista, cita, al hacer referencia a las relaciones entre femineidad y alimentación, trabajos que dan cuenta de la constitución temprana de patrones alimentarios diferentes en mujeres y varones. En un estudio de Brunet y Sezine (1966), citado por esta autora, se concluye que el $66 \%$ de las niñas recibieron alimentación de pecho en contraste con el $99 \%$ de los varones, las niñas fueron destetadas más tempranamente que los varones, y el tiempo dedicado a las actividades de alimentación con las niñas era significativamente menor. La misma discrepancia es observada en los patrones de sostenimiento en los brazos, tiempo que era más corto en el caso de las niñas. Fuera del cuestionamiento que podríamos hacer a las conclusiones que la autora hace a partir de estos estudios, abonando la desigualdad genérica, resulta interesante enfatizar la importancia de estos factores tempranos en la constitución de la identidad tanto en las mujeres como en los varones.

La relación entre los deseos de los lactantes y la respuesta que se da a ellos, configura patrones tempranos que pueden establecerse como núcleos de distorsión y malestar. Madres ansiosas que ofrecen sólo alimento - sea éste por pecho o por biberón-, frente a cualquier señal de disconformidad en el lactante, van confundiendo, retrasando y distorsionando el desarrollo psicofisiológico de las sensaciones de hambre y saciedad. Se sabe que estas alteraciones interoceptivas fundan algunos de los elementos diagnósticos fundamentales en la etiopatogenia de los trastornos alimentarios. El siguiente ejemplo ilustra esta idea:

Jessica, 7 años. Acude a la consulta con su madre. La niña presenta desde el nacimiento severos trastornos de la conducta alimentaria con negativa a ingerir sólidos. Se ha descartado patología del tracto gastrointestinal.
Tampoco se verifican alteraciones psicopatológicas de la serie psicótica que puedan explicar su rechazo a la comida. El desarrollo madurativo y emocional no presenta otros déficits y la integración social y, particularmente, escolar es adecuada a su edad cronológica. Su peso se encuentra ubicado en el extremo inferior de las tablas para su edad y altura y se alimenta exclusivamente con jugos y licuados. Madre e hija se embarcan desde hace 7 años en batallas por el poder alrededor del tema de la ingesta de comida. En estas luchas intervienen, también, otros miembros de la familia extensa. Cuanto más se niega Jessica a comer, más se involucran su madre y su abuela...

Desde su nacimiento, el niño va aprendiendo a regular sus estados internos, a chupar, a tragar y a establecer los límites del comienzo y terminación de su sesión de comida. Al mismo tiempo, va ofreciendo señales de hambre o plenitud. En la medida en que no pueda aprender a dominar estas señales adecuadamente o en la medida en que estas señales sean malinterpretadas por sus cuidadores, se sentarán las bases para el establecimiento de dificultades de apego y de individuación, de las cuales, seguramente, la conducta alimentaria será sólo un aspecto. Si en la lucha entre la autonomía y la dependencia, el niño o la niña quedan atrapados en la situación alimentaria, sus necesidades afectivas comenzarán a gobernar su conducta durante los momentos de alimentación, negándose a comer, por ejemplo. El $21 \%$ de la varianza del peso de niños entre los 12 y los 37 meses de edad puede ser explicado por variaciones en las medidas de temperamento (de los niños y sus madres) y conflicto alrededor de la alimentación. (Chatoor, Ganiban, Hirsch, Borman-Spurrell y Mrazek, 2000; Stein, Woolley y McPherson, 1999).

Más aún, podemos preguntarnos cuáles son los posibles efectos sobre la construcción de estructuras tempranas vincu- 
ladas a la alimentación que se generan en la interacción de madres afectadas con trastornos alimentarios y sus bebés. Otro trabajo del grupo de Stein (Stein, Woolley, Cooper y Fairburn, 1994) revela algunos resultados, cuanto menos interesantes. Las madres con problemas alimentarios parecen ser más intrusas en la interacción con sus bebés durante las horas de comida y también de juego, comparadas con las madres de un grupo de control. Manifiestan emociones más negativas y mayor nivel de conflicto durante las comidas. El tono emocional de sus bebés es más negativo y el peso de los mismos es tanto menor cuanto mayor es el nivel de conflicto puesto de manifiesto en la interacción, y cuanto mayores son las preocupaciones maternas acerca de su propio peso.

En esta línea de investigación se encuentran los estudios que intentan establecer la ocurrencia de alteraciones tempranas en los patrones alimentarios de los niños cuyas madres presentan antecedentes de, o cursan actualmente, trastornos alimentarios. En un trabajo de Agras, Hammer y McNicholas (1999), las bebés de madres con trastornos alimentarios chupaban significativamente más rápido y fueron destetadas 9 meses más tarde en comparación con las niñas de madres sin trastornos alimentarios. Además, se observó que las madres afectadas con este tipo de sintomatología alimentaban a sus hijas a intervalos más irregulares, usaban la comida con propósitos no alimentarios y estaban muy preocupadas por el peso de sus hijas. Los autores se preguntan acerca del riesgo del establecimiento de estos patrones tempranos para el desarrollo posterior de trastornos alimentarios.

La construcción de estructuras de significado de dominio, la tercera en la línea evolutiva, tiene que ver con la necesidad de ir otorgando significados a la experiencia respecto de los efectos diferen- ciales que tienen determinadas acciones sobre otras: algunas acciones serán conducentes a alguna forma de recompensa; otras, en cambio, conducirán a alguna forma de castigo. En palabras de Fernández-Álvarez (1992), «La sonrisa despierta ciertos gestos, la fiebre moviliza a los padres de cierta manera, la comida llega a ciertas horas y en cierta forma... Esta construcción muestra al self organizándose sobre la base de la necesidad de interpretar la experiencia de algún modo que le permita sobrevivir, la presencia de un esquema de poder que gobierna las relaciones en el mundo». Esta construcción gira en torno a principios de dominación y a la aparición de lo social en la forma de normas y sistemas de legalidad que regulan lo que puede y lo que no puede hacerse. El siguiente ejemplo tiene que ver con esta cuestión:

Celia, 18 años. Acude a la primera entrevista acompañada por su madre de 34. Difícil distinguir cuál era la madre y cuál la hija, no sólo por el aspecto físico, sino por el estilo caótico de funcionamiento. ¿Cómo ayudarlas a ambas a encontrar un orden que pueda comenzar a gobernar sus vidas? Para la madre, varios fracasos afectivos, dificultades laborales, el cuidado de su propia madre con demencia senil, y la imposibilidad de ejercitar su rol parental en el marco de una asimetría de poder con Celia. Para Celia, bulimia franca, abuso de alcohol, coqueteo con sustancias, conductas de riesgo y promiscuidad sexual.

Los patrones de interacción familiar entre miembros con características externalizadoras, como es el caso de esta viñeta, se expresan en serias dificultades de apego, de control de la impulsividad y de autonomía. Los intercambios muestran, a menudo, comunicaciones confusas en las que se entremezclan mensajes acusatorios con mensajes de pseudo-confirmación de la conducta problemática (Ratti, Humphrey y Lyons,1996). 
La construcción de la diferenciación sexual, relacionada con la discriminación de las diferencias entre los sexos y la gratificación corporal tanto activa como pasiva, permite comprender cómo operan los mecanismos de evolución psicosexual y la culminación de esta evolución en el drama edípico, entendido no sólo como un proceso intrapsíquico, sino como una forma de describir la particular interacción entre padres e hijos en un determinado momento histórico y cultural. Las experiencias de abuso sexual infantil intrafamiliar, por ejemplo, encontradas cada vez más entre los antecedentes de pacientes con trastornos alimentarios (Schwartz y Cohn, 1996), incidirán decisivamente en la configuración de estructuras de significados disfuncionales alrededor de los significados de gratificación sexual, por ejemplo.

La construcción de los significados de reconocimiento tiene que ver fundamentalmente con la capacidad para compartir con otros, para desarrollar conductas socialmente orientadas, no ya solamente en la esfera de lo familiar sino en grupos secundarios de pertenencia. Como ha escrito Fernández-Álvarez (1992): «La quinta construcción, la de reconocimiento, se organiza sobre la base de la necesidad de interpretar las diferentes exigencias del proceso de socialización que incluyen el cumplimiento de un desarrollo de actitudes, el manejo de sus emociones dentro de ciertos límites, la integración a un sistema de reglas extendido, la participación en grupos de pares, todo lo cual supone el despliegue de un repertorio de conductas del cual dependerá la posibilidad de ser reconocido en distintos ámbitos sociales.»

Procesos tempranos de descalificación y desconfirmación en la esfera de lo familiar, por ejemplo, incidirán negativamente en la construcción de significados de reconocimiento. La falta de validación de la propia experiencia genera situaciones de inseguridad y falta de confianza en las propias percepciones, sensaciones y sentimientos. El relato que sigue es un ejemplo de lo que se quiere decir:

Es difícil con mi papá... siempre está diciendo que tengo que ser razonable y aplicar mi criterio. Pero cada vez que traté de elegir algo por mi cuenta, incluso el libro que quería leer o el juego que quería jugar, él siempre tenía una idea mejor... Y yo terminaba aceptando... y lo malo es que terminaba convenciéndome de que sus elecciones eran mejores que las mías. Ahora estoy metida en esta relación y no sé cómo salir... No puedo afrontar el lío que se me viene si dejo a Luis... Se lleva tan bien con papá... (Lucía, 23 años)

En la adolescencia, las demandas evolutivas biológicas, psicológicas y sociales cobran una intensidad particular, que no se repite en ningún otro momento de la vida y que se expresa en todos los planos. En el biológico, a través del crecimiento físico, el aumento de la capacidad motora, la aparición de los caracteres sexuales secundarios y la maduración neurológica que posibilita el acceso al pensamiento operativo formal. La evolución conlleva también una serie de exigencias en lo social. En la adolescencia actual, los mandatos sociales son muy complejos, conformados por toda clase de expectativas de los adultos respecto al nivel de rendimiento académico, respecto de la posibilidad de conformar un proyecto vocacional o profesional, respecto de la presión ejercida por los grupos de pares, respecto de la presión social alrededor de determinados pautas, etc. En el plano del desarrollo emocional, los cambios no son menos significativos, ya que la capacidad expresiva y comunicativa que los adolescentes alcanzan en este período supone una serie de cambios profundos. Por eso pueden manifestarse en formas extremadamente pasionales, pueden ligarse con objetos trascendentes $\mathrm{y}$ fuera de su contexto inmediato. 
En la respuesta a todas estas demandas, el adolescente tendrá que, como culminación de la etapa, poder llegar a significar la experiencia de un modo autónomo. Autonomía que se habrá de reflejar en la posibilidad de desarrollar un guión personal. Los guiones (Fernández Álvarez, 1992) son esquemas, representaciones cognitivas que organizan la comprensión de las personas respecto de determinados eventos y guían el funcionamiento individual a través de un conjunto de acciones que tienen una extensión y una secuencia determinadas. Los guiones permiten a las personas transformar la complejidad del cúmulo de información que proviene del ambiente en niveles relativamente manejables $y$, al hacerlo, reducen la incertidumbre. Los guiones son personales, pero también pueden ser compartidos y presentar contenidos típicos de un grupo o una comunidad. Estas representaciones cognitivas muchas veces avanzan más allá de la información proporcionada por el ambiente y se constituyen operativamente en marcos de interpretación de la realidad y la experiencia de esa realidad. En este sentido, los guiones influyen activamente en las relaciones interpersonales y lo hacen definiendo roles y estableciendo cursos de acción. La elaboración de los guiones personales comienza de forma incipiente en la infancia. En cada ser humano, casi tan pronto como nace, se va personificando de un modo singular un programa constituido por el guión de los padres. Este proceso de encarnación del guión parental se vuelve particularmente significativo, en especial a partir de la pubertad, cuando se espera el desarrollo de las estructuras de autonomía, y que la persona se aboque a la tarea de construir su propio guión.

Una característica importante de los guiomes es su grado de flexibilidad o rigidez. Los guiones tienen, en ocasiones, un poder facilitador de los intercambios entre las personas cuando son compartidos o hay cierta consonancia y posibilidad de flexibilización. En otras ocasiones, por el contrario, tienen un poder obturador y de cierre, dificultan las relaciones entre las personas cuando son demasiado rígidos o demasiado discrepantes. Además, un guión personal que permita vivir una vida satisfactoria tendrá que tener, por un lado, cierto grado de concordancia y, por otro, cierto grado de discrepancia con relación al guión paterno. Construir un guión personal viable implica una tarea constructiva a partir de la integración de elementos previos tomados del guión parental, y una tarea de separación, en mayor o menor grado, de ese guión paterno que lo antecede. En las consultas de los adolescentes y/o su familia, observaremos siempre algún obstáculo a esta tarea, expresada en algún tipo de sufrimiento o malestar. Este padecimiento traduce alguna imposibilidad de articular adecuadamente los mandatos precedentes (paternos) y el propio guión adolescente. Los obstáculos en el desarrollo del guión personal pueden ir de leves a severos, y su afectación se hace evidente en múltiples situaciones clínicas.

Los estudios vinculados al papel que las dificultades en el funcionamiento autónomo juegan en la etiopatogenia de los trastornos alimentarios son numerosísimos. Estas dificultades fueron identificadas tempranamente en la literatura sobre el tema. Algunos de tales estudios utilizan medidas de dependencia afectiva y excesiva orientación hacia los demás (sociotropía) (Friedman y Whisman, 1998; Narduzzi y Jackson, 2000). Los procesos de individuación/separación han sido precozmente objeto de análisis por su vinculación con diversas problemáticas psicológicas y trastornos psiquiátricos. La excesiva adherencia y el perfeccionismo, rasgos que aparecen con suma frecuencia en la narrativa de mujeres 
afectadas de trastornos alimentarios (como el caso de Lucía citado anteriormente), constituyen otro aspecto de la problemática del reconocimiento y la autonomía en la génesis y mantenimiento de trastornos alimentarios, particularmente en momentos de aumento de las demandas evolutivas (Wechselblatt, Gurnick y Simon, 2000).

\section{MÁS ALLÁ DE LA AUTONOMÍA}

La evolución de las estructuras de significado no se detiene, por supuesto, con la construcción de significados de autonomía. Muchas demandas evolutivas se plantean todavía una vez cumplido ese período. La tarea de significación de la experiencia no se detiene nunca, nos acompaña a lo largo de nuestra vida como adultos y más allá aún cuando en la ancianidad nos enfrentamos con la tarea de darle un significado a la finitud de la existencia.

No nos extenderemos en el desarrollo de cómo se construyen las estructuras de significado de la transitividad, la perdurabilidad, la productividad y el recogimiento. No obstante, en la medida en que los trastornos alimentarios permanezcan en el tiempo, se amplía el espectro de presentación de los mismos a otras edades, más allá de la adolescencia. Es importante tener en cuenta cómo se afectan estas estructuras más tardías en individuos adultos que padecen alguna forma de malestar vinculada con el desarrollo de trastornos alimentarios. Todos los órdenes de su experiencia se ven afectados: su vida afectiva, su productividad laboral, el ejercicio de los roles familiares, etc. Ya hemos mencionado anteriormente algunos estudios vinculados a la influencia que las actitudes maternas de mujeres afectadas con distinto tipo de problemática alimentaria tienen en la constitución de patrones tempranos de relación con sus hijos. Las siguientes viñetas clínicas ilustran las presentaciones de esta problemática más allá de las típicas de la adolescencia:

Jorge, 37 años, ingeniero de sistemas. Su vida, como él mismo la describe es «una carrera". Refiere que 'entrena' desde los 20 años, desde que una amiga ocasional le dijo que lo veía "rellenito y flojo». La progresión de su actividad física fue escalando desde su inicio. Lo que podía entenderse, al principio, como un interés deportivo en el que encontraba incluso cierto alivio y gratificación, fue transformándose en algo obsesivo y perturbador que terminó afectando todos los aspectos de su vida. Los kilómetros que corre o anda en bicicleta aumentan, las horas "de fierros" se prolongan, las dietas 'saludables' se hacen cada vez más estrictas, determinados alimentos van desapareciendo de sus preferencias. Toda una parafernalia de vitaminas, aminoácidos, minerales, reconstituyentes va poblando su botiquín. No lo detienen ni la ocurrencia de varios desgarros musculares, ni una fractura en el pie, ni una dislocación de la articulación escápulo-humeral. Jorge proviene de una familia orientada hacia el logro, ambos padres son profesionales, al igual que sus hermanos. Una de sus primeras imágenes en sesión tenía que ver con él sintiéndose como en una pecera, con sus padres, sus tíos y sus hermanos mayores observándolo y comentando su proceder. Su vida profesional también es una carrera, las mejores notas en la primaria, ni un solo fracaso en la secundaria, su licenciatura a los 21 años, un master a los 24 , etc... etc... Va por el segundo matrimonio y haciendo agua... Tiene 5 hijos repartidos entre ambos: con ellos se siente un extraño. Sus "obsesiones» comienzan a afectar su trabajo. Esto lo trae a la consulta...

Alicia, 32 años. Recuerda que durante toda su vida hizo dieta para pelear contra un peso empeñado en salirse de los estándares sociales. Pero no desarrolla sintomatología hasta su primer embarazo, a los 27 años, en que comienza a vomitar y a tener severos episodios de atracón. Su bulimia continúa durante los últimos 5 años, entremezclándose con sus preocupaciones por el cuidado de su bebé, las importantes demandas de su trabajo les secre- 
taria ejecutiva en una empresa petrolera), y la construcción de una nueva casa, proyecto que pasa a monopolizar todos los intercambios de la pareja. Un nuevo embarazo la trae a la consulta. Este coincide con la culminación del ansiado proyecto familiar y la mudanza. La sintomatología se recrudece...

Algunos trabajos demuestran que durante el embarazo los síntomas alimentarios se comportan de diferente manera. Existen observaciones que dan cuenta de su mejoría en los primeros meses y de su empeoramiento durante los últimos (Fairburn, Stein y Jones, 1992). Las mujeres con trastornos alimentarios tienden a dar a luz niños pequeños para la edad gestacional y esto se correlaciona con bajo peso materno previo al embarazo, tabaquismo, baja ganancia de peso semana a semana y elevaciones en las escalas de bulimia (Conti, Abraham y Taylor, 1998).

Comenzábamos este trabajo con la caracterización de la naturaleza múltiple y compleja de los problemas relacionados con la conducta alimentaria. Así como en muchas otras situaciones clínicas, los trastornos alimentarios requieren del despliegue de una gran diversidad de técnicas y abordajes, de recursos y habilidades. Pensamos que la mayor riqueza de nuestro modelo tiene que ver, por una parte, con la conceptualización y la práctica de la psicoterapia. La práctica de la psicoterapia, para nosotros, implica cierto cambio, y este cambio tiene que ver con que las personas puedan hacer algo con los significados, puedan movilizar recursos psicológicos para producir cambios en los significados que sostienen la experiencia de sufrimiento. Por otra parte, la psicoterapia es una cuestión de lenguaje -en sentido amplio- en el marco de un proceso interactivo. No importa que se trate de una sola persona o de familias, los significados van a ser diferentes y también los lenguajes pero, en esencia, alude a un mismo proceso que se traduce en una interacción.
Una de las ventajas que a nuestro entender posee nuestro modelo tiene que ver con que cada situación de malestar o sufrimiento es, en sí misma, única. $\mathrm{Si}$ bien puede compartir características similares con otras, el diseño de las estrategias de tratamiento destinadas a poner en marcha los procesos de cambio necesarios debe ser hecha a medida. Los recursos con los que contamos, pacientes y equipo terapéutico, son como ingredientes de una receta. Importa la cualidad de los ingredientes, importan las cantidades, importa el orden de incorporación de cada uno de ellos al proceso. Importa la habilidad para identificar estas pertinencias ¿qué, cuánto, en qué momento? Importa la creatividad para afrontar aquellas situaciones en que alguno de los ingredientes falta. $\mathrm{Y}_{¿ \text { Cuáles }}$ son los ingredientes que no pueden faltar? La respuesta a esta pregunta es una buena excusa para otro trabajo.

\section{REFERENCIAS BIBLIOGRÁFICAS}

Agras, S., Hammer, L., y McNicholas, F. (1999). A prospective study of the influence of eating-disordered mothers on their children. International Journal of Eating Disorders 25, 253-262.

Chatoor, I., Ganiban, J., Hirsch, R., BormanSpurrell, E., y Mrazek, D.A. (2000). Maternal Characteristics and Toddler Temperament in Infantile Anorexia. American Academy of Child and Adolescent Psychiatry, 39, 743-751.

Conti, J., Abraham, S., y Taylor, A. (1998). Eating behavior and pregnancy outcome. Journal of Psychosomatic Research, 44, 465-477.

Fairburn, C.G., Stein, A., y Jones, R. (1992). Eating habits and eating disorders during pregnancy. Psychosomatic Medicine, 54, 665-672.

Fernández-Álvarez, H. (1992). Fundamentos de un modelo integrativo en psicoterapia. Buenos Aires: Paidós. 
Friedman, M.A., y Whisman, M.A. (1998). Sociotropy, autonomy, and bulimic symptomatology. International Journal of Eating Disorders, 23, 439-442.

- Narduzzi, K.J., y Jackson, T. (2000). Personality differences between eating-disordered women and a nonclinical comparison sample: a discriminant classification analysis. Journal of Clinical Psychology, 56, 699-710.

Orbach, S. (1985). Accepting the Symptom: A Feminist Psychoanalytic Treatment of Anorexia Nervosa. En: David M. Garner y Paul E. Garfinkel (Eds.), Handbook of Psychotherapy for Anorexia Nervosa and Bulimia. Nueva York: Guilford Press.

Ratti, L.A., Humphrey, L.L., y Lyons, J.S. (1996). Structural analysis of families with a polydrug-dependent, bulimic, or normal adolescent daughter. Journal of
Consultant and Clinical Psychology, 64, 1255-1262.

Schwartz, M.F., y Cohn, L. (Eds.) (1996). Sexual Abuse and Eating Disorders. Bristol: Brunner/Mazel.

Stein, A., Woolley, H., Cooper, S.D., y Fairburn, C.G. (1994). An observational study of mothers with eating disorders and their infants. Journal of Child Psychology and Psychiatry, 35, 733-748.

Stein, A., Woolley, H., y McPherson, K. (1999). Conflict between mothers with eating disorders and their infants during mealtimes. British Journal of Psychiatry, $175,455-461$.

Wechselblatt, T., Gurnick, G., y Simon, R. (2000). Autonomy and relatedness in the development of anorexia nervosa: a clinical case series using grounded theory. Bulletin of the Menninger Clinic, 64, 91-123. 